\title{
Influência da propaganda na utilização de medicamentos em um grupo de idosos atendidos em uma unidade básica de saúde em Aracaju (SE, Brasil)
}

\section{The influence of the advertising in the medication use in a group of elderly attended in a primary health care unit in Aracaju (Sergipe, Brasil)}

\author{
Divaldo Pereira deLyra Jr. ${ }^{1}$ \\ Aline Souza N eves ${ }^{1}$ \\ KarineSantos Cerqueira ${ }^{1}$ \\ Paulo Sergio $M$ arcellini ${ }^{1}$ \\ Tatiane Cristina $M$ arques ${ }^{2}$ \\ JoséAugusto Cabral deBarros ${ }^{3}$
}

\footnotetext{
${ }^{1}$ Laboratório deEnsino e Pesquisa em Farmácia Social, Departamento de Fisiologia, Universidade Federal de Sergipe. Cidade Universitária Professor JoséAloísio de Campos. Av. $M$ arechal Rondon $s / n^{\circ}$, Jardim Rosa Elze. 49100-000 São Cristóvão SE. Iyra_jr@hotmail.com ${ }^{2}$ Departamento de Enfermagem Geral e Especializada da Escola de Enfermagem de Ribeirão Preto, Universidade de São Paulo.

${ }^{3}$ Departamento deSaúde Coletiva, Faculdade de M edicina, Universidade Federal de Juiz deFora.
}

Abstract The growth of the Brazilian elderly people has led to a trend to an increase in the medication use. The inadequate use of drugs can be induced by some factors, like advertisement, with the risk of damaging the user's health. The objective of the study was to evaluate the advertisement influence in medication use in a group of elderly patients in a primary health care unit in A racaju, Sergipe, Brazil. 230 elderly aging over 60 years with both genders had been interviewed from A pril to June of 2007. The majority of the interviewees (73\%) has at least a chronic health condition and $73.9 \%$ consumed regularly at least one medication. $17.8 \%$ of the sample informed to use medication moti vated by publicity influence; $2.2 \%$ had considered that the medication never cause damages and $6.5 \%$ believed that always it makes well. In this study, correlations have been made and demonstrated that those who presented a higher level of consumption influenced by advertising also think that drugs used are always beneficial and vice versa $(p=0.04)$. The data showed that part of elderly suffered influence of advertisement to medication use, and arenot conscious of risks involved.

Key words Elderly, Medication advertisement, Medication use
Resumo 0 crescimento da população idosa brasileira tem levado a uma maior tendência ao uso de medicamentos. A sua utilização inadequada pode ser induzida por vários fatores, dentre eles a propaganda, podendo trazer danos à saúde do usuário. 0 objetivo do estudo foi avaliar a influência da propaganda no consumo de medicamentos por um grupo de idosos atendidos em unidade básica desaúdedeAracaju, Sergipe. No período deabril a junho de 2007, um grupo de230 idosos, com idade acima de 60 anos, de ambos os gêneros, foi entrevistado. A maioria dos entrevistados (73\%) possuía pelo menos uma doença crônica e $73,9 \%$ consumiam pelo menosum medicamento regularmente. Do total de entrevistados, $17,8 \%$ da amostra relataram utilizar medicamentos por influência da propaganda; $2,2 \%$ consideraram que os medicamentos veiculados na mídia nunca fazem mal e $6,5 \%$ acreditavam que eles semprefazem bem. No estudo, correlações foram feitas e demonstraram quequem mais consomemedicamentosinfluenciados pela propaganda também considera que eles sempre fazem bem e vice-versa $(p=0,04)$. 0 s dados revelaram que parte dos idosos sofreu influência da propaganda no consumo de medicamentos, não levando em consideração os riscos que estes poderiam causar.

Palavras-chave Idosos, Propaganda de medicamentos, U so de medicamentos 
Introdução

Atualmente, o crescimento da população deidosos, em números absolutos e relativos, está ocorrendo em um nível sem precedentes na história da humanidade. Em 1950, eram cerca de $204 \mathrm{mi}$ Ihões deidosos no mundo, ejá em 1998 este contingente alcançou 579 milhões de pessoas; um crescimento de quase oito milhões de idosos por ano. Estima-se que em 2050 as pessoas com mais de 60 anos representarão $32 \%$ da população mundial esuperará pela primeira vez na história o número de crianças $^{1-3}$.

No Brasil, a vida média vem sofrendo notável incremento, tendo passado de 1940 aos dias de hoje por um aumento de $60 \%$ (de 62,6 anos, em 1980, para 71,7, em 2004), com projeções de que até 2025 chegará aos 80 anos. Segundo os dados do Instituto Brasileiro de Geografia e Estatística (IBGE) para 2002, osidososjárepresentavam 9,3\% (16,22 milhões que, em 2005, já haviam ascendido a 18 milhões) da população, estimando-seque em 2002 seriam 11,4\% dos 219 milhões de brasileiros, sendo esperado que cheguem a 35 milhões até2050. ${ }^{4}$ Esse crescimento colocaráo país na condição de detentor da sexta maior população de idosos do mundo, em termos absolutos s.6. Como $^{5}$ consequência, ocorre uma grande preocupação para a Saúde Pública, pois com o aumento da idadecronológica há maior prevalência decondições crônicas de saúde, apresentando assim uma tendência ao uso de medicamentos.

Os dados apontados indicam vir o país passando por uma transição demográfica e epidemiológica bastantecomplexa, com a convivência de doenças não transmissíveis ou produzidas pelo homem, com doenças infecciosas e parasitárias com variável prevalência delas, conforme as macrorregiões que seconsiderem, além decrescentee preocupanteaumento da mortalidade por causas externas, em especial a violência, sobretudo em segmentos mais jovens da população. ${ }^{7}$

As alterações farmacocinéticas afetam a concentração dos fármacos em seus sítios de ação, e as de caráter farmacodinâmico se associam às diferenças de resposta do organismo a uma determinada concentração do princípio ativo. 0 processo de envelhecimento explica os fenômenos apontados, já que, nos idosos, há uma diminuição da massa muscular e da água corporal, com possível alteração da biodisponibilidade de alguns fármacos; a filtração e a excreção renal, assim como o metabolismo hepático e mecanismos homeostáticos, podem ficar comprometidos, o que pode acarretar obstáculos à elimina- ção de metabólitos, levando a reações adversas ${ }^{8-}$ ${ }^{12}$. No entanto, as peculiaridades das enfermidadescrônico-degenerativasfavorecem a prescrição e o uso de múltiplos medicamentos e, em consequência, de riscos aumentados das interações ou dos efeitos indesejáveis aos quais podem estar associados gastos desnecessários e internações hospitalares que poderiam ter sido evitadas.

0 emprego do arsenal terapêutico por idosos apresenta uma linha tênue entreo benefício e o risco, ou seja, se por um lado ajuda a estender a longevidade, por outro pode afetar negativamente a sua qualidade de vida. Logo, o problema não pode ser atribuído ao consumo de medicamentos, mas sim à irracionalidade de seu uso, que expõe os idosos a riscos potenciais $s^{13,14}$.

Segundo Teixeira e Lefèvre ${ }^{9}$, a partir da última década, o uso inapropriado dos medicamentos por idosos tem setornado um problema tanto do ponto de vista clínico quanto do econômico. Nos Estados Unidos, por exemplo, os custos com a morbimortalidade associada aos medicamentos triplicaram nos últimos anos, sendo que somente osidosos gastaram US $\$ 104$ bilhões, bem mais do que foi gasto com qualquer outro problema de saúde nessa faixa etária ${ }^{13,15}$. Na Espanha, os problemas associados à farmacoterapia foram responsáveis por aproximadamente $5 \%$ dos ingressos hospitalares, com um custo médio de 3.000 euros por internação ${ }^{16}$.

No Brasil, o consumo de especialidades farmacêuticas, principalmente entre osidosos, éfavorecido pela multiplicidade de produtos lançados no mercado, pela publicidade que os cerca e pela simbolização da saúde que o medicamento pode representar ${ }^{17,18}$. Outro aspecto importante diz respeito à indefinição do perfil de utilização de medicamentos nessa faixa etária, pois poucos estudos são encontrados na literatura e, em sua mai oria, concentram-senas regiões Sul e Sudeste do Brasi ${ }^{12,19}$. Aliada a esses fatores, a ausência do farmacêutico na farmácia também resulta em estabelecimentos desassistidos e banaliza a figura do medicamento, que passa simbolicamente a representar um bem de consumo e não um instrumento de saúde ${ }^{20}$.

A exploração do valor simbólico do medicamento - socialmente sustentado pela indústria farmacêutica, por agências de publicidade e empresas de comunicação - passa a representar um dos mais poderosos instrumentos para a indução e o fortalecimento de hábi tos voltados para 0 aumento de seu consumo ${ }^{21}$. Esse fenômeno ocorreem um contexto deuma sociedadena qual a ideologia de consumo, associada à visão meca- 
nicista e biomédica do processo saúde/doença e à lógica de mercado, termina por levar a uma hipervalorização do papel do medicamento e à intensificação da "medicalização". As indústrias farmacêuticas, por exemplo, gastam em média $35 \%$ do valor das vendas com a chamada "promoção farmacêutica", publicidadee marketing de seus produtos ${ }^{22}$. N esse contexto, o impacto da propaganda de medicamentos e demais produtos relacionados à saúdeinfluenciam tanto a prática dos profissionais do setor quanto às demandas das populações.

De acordo com a Agência Nacional de Vigilância Sanitária (Anvisa) ${ }^{23}$, a propaganda é um método queestimula o consumo de medicamentos, pois engloba o conjunto de técnicas utilizadas para divulgar conhecimentos e/ou promover adesão a princípios, ideias ou teorias, visando exercer influência sobre o público. Além disso, a publicidade de medicamentos definepadrões de mercado e de comportamento das pessoas, exercendo impacto concreto sobre as práticas terapêuticas ${ }^{24}$. Logo, a preocupação com a qualidade da informação disponível sobre o medicamento deve fazer parte do cotidiano dos profissionais de saúde e dos consumidores ${ }^{25}$.

$N$ as últimas três décadas, pesquisadores como Lexchin ${ }^{26}, \mathrm{H}$ erxheimer et al. ${ }^{27}$, Silverman et al. ${ }^{28}$, Avorn ${ }^{29}$ e, no Brasil, Barros ${ }^{30}$, Pizzol et al. ${ }^{31}$ e Fagundes et al. ${ }^{32}$ têm publicado estudos sobreo tema, alertando para as consequências das relações entre profissionais da saúde e indústria farmacêutica. Entretanto, énecessário desenvolver maispesquisas que avaliem o impacto da propaganda no consumo degrandenúmero deespecialidades farmacêuticas disponíveis no mercado. Baseado no exposto, o presente estudo teve como meta avaliar a influência da propaganda no consumo de medicamentos em um grupo de idosos assistidos por uma unidadebásica de saúdeem Aracaju (SE).

\section{Metodologia}

\section{Desenho do estudo}

Foi realizado um estudo analítico, transversal, com amostragem por conveniência, em uma unidade básica de saúde do município de Aracaju (SE), de abril a junho de 2007.

Local de estudo

O estudo foi realizado na Unidade Básica de Saúde (UBS) Edézio Vieira de M elo, do bairro Siqueira Campos, pertencente à Secretaria Mu- nicipal deSaúde(SM S) deAracaju. 0 centro comporta o H ospital Dia e o Centro de Reabilitação Física e M otora de Aracaju, contan do com cerca de 70 profissionais de saúde de sete diferentes especialidades, como ginecologia e odontologia.

População e amostra do estudo

A referida U BS atende 19.000 usuários cadastrados e residentes no bai rro Siqueira Campos. Participou do estudo um grupo de 230 usuários com idade acima de 60 anos, de ambos os gêneros, residentes edomiciliados em Aracaju, cadastrados na UBS. Os idosos foram selecionados pelos agentes comunitários de saúde da UBS, e os convites, bem como as entrevistas, foram feitos nas visitas domiciliares realizadas pelos pesquisadores.

\section{Coleta dos dados}

Os dados foram coletados por meio de entrevistas individuais utilizando um questionário semiestruturado, contendo as variáveis sociodemográficas: gênero, escolaridade (em anos completos), renda familiar (em salários mínimos), idade (em anos completos) e ocupação, bem como variáveis relativas à propaganda (utilização de medicamentos veiculados na mídia, opinião sobre a eficácia dos medicamentos anunciados e acesso das propagandas de medicamentos nos meios de comunicação) e consumo de medicamentos ("qual a causa/indicação do uso do(s) medicamento(s)" e "qual(is) medicamento(s) em uso"). No estudo, os medicamentos foram classificados segundo o nome genérico ou demarca. As entrevistas foram realizadas somente após a assinatura do Termo de Consentimento Livre eEsclarecido.

Foram incluídos no banco de dados todos os medicamentos industrializados, assim como as fórmulas magistrais. Foram excluídos os medicamentos homeopáticos, os florais de Bach, chás, decoctos, tinturas e aqueles que não possuíam formulação clara33.

\section{Análise dos dados}

Os dados coletados foram reunidos em um banco de dados e analisados pelo programa estatístico Epi Info versão 3.4. Além da estatística descritiva, foram realizados testes de associação de qui-quadrado para avaliar as correlações entre as variáveis, sendo considerado como significantep $<0,05$. 
Foi considerado alfabetizado funcional, de acordo com a Unesco, o indivíduo capaz de utilizar a leitura e a escrita para fazer frente às demandas de seu contexto social e de usar essas habilidades para continuar aprendendo e se desenvolvendo ao longo da vida ${ }^{34}$.

Para identificar as substâncias a partir dos nomes comerciais, empregou-se o Dicionário Terapêutico Guanabara 2005/2006. Após a identificação, os medicamentos foram classificados deacordo com a Anatomical Therapeutical Classification (ATC).

\section{Aspectos éticos}

O projeto foi aprovado pelo Comitê de Ética em Pesquisa do Hospital Universitário da Universidade Federal de Sergipe.

\section{Resultados ediscussão}

No presente estudo, os resultados referentes às variáveis sociodemográficas estão demonstrados na Tabela 1.

A partir da análise dos dados foi verificado que, dos 230 entrevistados, 31,3\% estavam na faixa etária acima de 75 anos, achados semelhantes aos encontrados na literatura. Tais dados confirmam o crescimento considerável do contingente populacional de brasileiros com mais de 60 anos $^{35,36}$.

Quanto ao gênero, 72,6\% da amostra foi composta por mulheres. Este resultado é semeIhante a estudos prévios que atribuem maior expectativa de vida à mulher em_relação ao homem no Brasil. Isso é explicado pela proteção cardiovascular gerada pel os hormônios femininos, descenso nas taxas de mortalidade materna, menor exposição a fatores de risco como tabaco e álcool, além da maior procura por assistência médica, em virtudedeuma percepção mais acentuada das doenças ${ }^{37,38,39}$.

No estudo, mais da metade dos idosos cursou ensino fundamental e 10\% foram considerados analfabetos; os dados são condizentes aos encontrados na literatura. Entre 1995 e2005 houve redução no número de analfabetos no país, todavia as desigual dades regionais continuam muito acentuadas ${ }^{40}$. No que concerne à ocupação, a maioria dos entrevistados foi composta por aposentados (73,9\%), com renda que varia de um a três salários mínimos (88,3\%). Esses dados corroboram os apresentados pelo IBGE ${ }^{40}$, que identificou $65,3 \%$ de idosos aposentados no Brasil,
Tabela 1. Distribuição da população de estudo segundo idade, gênero, escolaridade, ocupação e renda na UBS Edézio Vieira (Aracaju, SE) - abril a junho de 2007.

\begin{tabular}{lrr}
\hline Variáveis sociodemográficas & $\mathrm{f}$ & $\mathrm{f} \%$ \\
\hline Idade & & \\
$60-65$ & 46 & 20 \\
$65-70$ & 51 & 22,2 \\
$70-75$ & 61 & 26,6 \\
$>75$ & 72 & 31,3 \\
Gênero & & \\
Feminino & 167 & 72,6 \\
M asculino & 63 & 27,4 \\
Escolaridade & & \\
Ensino superior & 5 & 2,2 \\
Analfabeto & 23 & 10 \\
Alfabetizado & 33 & 14,4 \\
Ensino médio & 36 & 15,6 \\
Ensino fundamental & 133 & 57,8 \\
Ocupação & & \\
Autônomo & 4 & 1,7 \\
Empregado & 4 & 1,7 \\
Dona de casa & 21 & 9,1 \\
Outros & 31 & 13,5 \\
Aposentado & 170 & 73,9 \\
Renda & & \\
6 - 10 & 9 & 3,9 \\
3 - 6 & 18 & 7,8 \\
1 - 3 & 203 & 88,3 \\
Total & 230 & 100 \\
\hline
\end{tabular}

sendo esse número maior na região Nordeste, com $72,2 \%$.

Devido ao maior envelhecimento, foi verificado que $73 \%$ dos entrevistados possuem pelo menos uma condição crônica de saúde, sendo as mais frequentes as do sistema cardiovascular (54,3\%), musculoesquelético ( $22,7 \%$ ) eendócrino $(12,1 \%)$ (Tabela 2). Dentre elas, as mais prevalentes foram a hipertensão arterial sistêmica (145 casos) e o diabetes (48 casos). De acordo com Vivian ${ }^{41}$, a associação entre essas doenças é bastantegrave, pois multiplica o risco de complicações decorrentes de ambas as situações quando avaliadas isoladamente.

No estudo, foi verificado que $73,9 \%$ dos entrevistados consomem pelo menos um medicamento regularmente. Este dado também fica evidenciado na literatura nacional e internacional, pois a grande maioria dos idosos utiliza no mínimo um medicamento diariamente, 10,41. Quanto às classes terapêuticas, as mais utilizadas foram: 
inibidores da enzima conversora da angiotensina (20\%), antiinflamatóriosnão esteroidais ( $14,8 \%$ ), diuréticos (13\%) ehipoglicemiantes (10,2\%) (Tabela 3). 0 uso dessas classes terapêuticas está associado à alta prevalência de condições crônicas de saúde, características da faixa etária ${ }^{10}$.

No que tange à questão de os medicamentos destinados aos problemas cardiovasculares serem os mais utilizados pelos idosos neste estudo, os resultados foram similares aos já descritos na literatura ${ }^{37,42}$. Tal perfil de consumo pode ser justificado pelo fato de as doenças cardiovasculares liderarem as cau sas de morbimortalidade em indivíduos com idade acima de 65 anos $^{42}$. Por sua vez, $26,5 \%$ dos idosos, por ocasião da entrevista, relataram não utilizar medicamentos. Este acha-

Tabela 2. Distribuição de frequência(f) dos problemas de saúde apresentados pela população de estudo, segundo a ATC, na UBS Edézio Vieira (Aracaju, SE) - abril a junho de 2007.

\begin{tabular}{|c|c|c|}
\hline Problemas de saúde & $f$ & $f \%$ \\
\hline Sistema cardiovascular * & 253 & 54,3 \\
\hline Sistema musculoesquelético & 106 & 22 \\
\hline Sistema endócrino & 56 & 12,1 \\
\hline Órgãos sensoriais ** & 24 & 5 \\
\hline Sistema nervoso & 19 & 4 \\
\hline Sistema respiratório & 8 & 1, \\
\hline Total & 466 & 100 \\
\hline
\end{tabular}

*Angina, insuficiência cardíaca congestiva, hipertensão arterial sistêmica; ** Visão, audição.

Tabela 3. Distribuição de frequência e classificação dos medicamentos utilizados pela população de estudo, segundo a ATC, na UBS Edézio Vieira (Aracaju, SE) - abril a junho de 2007.

\begin{tabular}{lrr}
\hline \multicolumn{1}{c}{ Classe terapêutica } & \multicolumn{1}{c}{$\mathrm{f}$} & $\mathrm{f} \%$ \\
\hline Anti-inflamatórios não esteroidais & 74 & 14,8 \\
Inibidores da enzima conversora da & 100 & 20 \\
angiotensina & & \\
Diuréticos & 65 & 13 \\
Hipoglicemiantes & 51 & 10,2 \\
Betabloqueadores & 30 & 6 \\
Bloqueadores de canal de cálcio & 20 & 4 \\
Cardiotônicos & 10 & 2 \\
Psicolépticos & 12 & 2,4 \\
Outros & 137 & 27,6 \\
Total & 499 & 100 \\
\hline
\end{tabular}

do pode ser confirmado por outros estudos realizados no Brasil, em que a proporção de idosos que não faz uso de qualquer farmacoterapia é de $4 \%$ a $10 \%$, mas pode chegar a $20 \%$ ou mais ${ }^{10}$.

Com relação à propaganda, os resultados demonstraram que $17,8 \%$ da amostra relataram utilizar medicamentos influenciados por ela. Levantamentos feitos na população brasileira mostraram que, no Recife (PE), 2,6\% dos medicamentos foram adquiridos pelo efeito de propaganda, dado maior do que os encontrados nas cidades de Ribeirão Preto (SP) eAraraquara(SP), em que a influência foi relativamente pequena, $1,1 \%$, e $0,2 \%$, respectivamente ${ }^{43-45}$. Porém, um estudo mais recente identificou que $31 \%$ da população entrevistada comprou medicamentos sob influência de propaganda ${ }^{46}$.

Vale ressaltar que, além do consumidor, as propagandas também são planejadas para atingir desde o médico e o farmacêutico até o dono da farmácia, o balconista e o paciente, conseguindo influenciar a prescrição, a dispensação, a venda e o consumo demedicamentos, sendo considerada ainda como atualização terapêutica pelos profissionais de saúde ${ }^{47}$. A mídia é vista como uma poderosa ferramenta, capaz de "motivar a demanda pelo consumidor final, formar opinião entre os que prescrevem e exercer pressão sobre as políticas públicas" ${ }^{48}$. Rollason et al. ${ }^{49}$ citam que $50 \%$ dos pacientes, em especial osidosos, que vão ao médico esperam a indicação de um medicamento, e os dados demonstram que $75 \%$ dessas consultas resultam em prescrição.

No presente estudo, $33,5 \%$ e $28,3 \%$ consideraram quea propaganda deveser limitada e proibida, respectivamente (Tabela 4).

Tabela 4. Distribuição de frequência quanto ao acesso às propagandas de medicamentos nos meios de comunicação na UBS Edézio Vieira (Aracaju, SE) - abril a junho de 2007.

\begin{tabular}{lrr}
\hline \multicolumn{1}{c}{$\begin{array}{c}\text { Acesso às propagandas } \\
\text { de medicamentos }\end{array}$} & $\mathrm{f}$ & $\mathrm{f} \%$ \\
\hline Livre & & \\
Limitada & 79 & 34,3 \\
Proibida & 77 & 33,5 \\
N ão opinaram & 65 & 28,3 \\
Total & 9 & 3,9 \\
& 230 & 100 \\
\hline
\end{tabular}


Nascimento e Sayd ${ }^{21}$ afirmam: A propaganda de medicamentos realizada hoje no Brasil está em clara contradição com a atual Política Nacional de M edicamentos (1998), segundo a qual o uso de produtos farmacêuticos deve se dar de forma racional, ética e correta, preconizando explicitamente um maior "controle da propaganda dos medicamentos de venda livre". Entretanto, os primeiros passos para a limitação dessas propagandas estão sendo dados, mesmo que lentamente e de forma pouco eficaz. Por exemplo, embora no final das propagandas seja obrigada a inserção da frase: "Ao persistirem os sintomas, o médico deverá ser consultado", esta indica primeiramente o consumo irracional, incorreto ou inconsciente de medicamentos e só depois a busca pelo profissional de saúde ${ }^{21}$, diferente das propagandas na Espanha, que afirmam: "Leia as instruções deste medicamento e consulte o farmacêutico". Para 34,3\% dos entrevistados, a propaganda de medicamentos deveser livre. Lucchese et al. ${ }^{50} \mathrm{ob}$ servaram que a propaganda estimula e/ou induz o uso indiscriminado de medicamentos.

Outros achados interessantes mostraram que $8,7 \%$ dos entrevistados consideraram que os medicamentos veiculados na mídia não trazem qualquer dano à saúde. Esses resultados preocupam, pois em uma avaliação das peças publicitárias monitoradas pela Anvisa, Heineck et al. ${ }^{51}$ encontraram mais de $80 \%$ de peças com infrações à legislação sanitária, com omissão de informações fundamentais a respeito de cuidados, reações adversas e contraindicações. Frosch et al. . $^{52}$, nos Estados Unidos, constataram que as propagandas de medicamentos oferecem limitada informação sobre precauções. Segundo Le fèvre $\mathrm{e}^{20}$, os medicamentos conseguem, muitas vezes, funcionar eficazmente como símbolos de saúde, pois "iludem os indivíduos com a aparência de eficácia científica". Portanto, é perfeitamente possível ver o medicamento como um signo cujo significado vai bem mais além da simples ideia, conceito imagem mental de "saúde", implicando também, e, sobretudo, a própria realização ou obtenção dela.

No presente estudo, foram realizadas correlações empregando as variáveis: utilização de medicamentos veiculados na mídia eopinião sobrea eficácia dos medicamentos anunciados. Tal análise demonstrou que quem mais consumiu medicamentos influenciado pela propaganda também considerou que eles sempre fazem bem e vice-versa $(p=0,04)$. Bertoldi et al. ${ }^{53}$ afirmam que a insatisfação com a saúde, além de determinar o uso de medicamentos por fatores farma- cológicos que se relacionam a uma necessidade real de utilização, é motivadora de fatores culturais e comportamentais que resultam em um aumento desse uso. Uma das causas desse tipo de utilização se refere à função simbólica dos medicamentos, isto é, o mito de que eles resolvem qualquer problema ${ }^{10}$.

Dos entrevistados, $64,3 \%$ acreditam que os medicamentos anunciados às vezes podem fazer mal. Outro estudo mostra que alguns idosos se tornam tão ligados simbolicamente aos medicamentos que reconhecem a necessidade deles em suas vidas, pois são como um "mal necessário", e muitos acreditam que se não tomarem os medicamentos corretamente vão se sentir mal ${ }^{54}$.

Outra correlação utilizando as variáveis utilização de medicamentos veiculados na mídia e acesso às propagandas de medicamentos nos meios de comunicação demonstrou que quem consumiu maismedicamentosinfluenciados pela mídia também foi favorável à liberação das propagandas, sem qualquer controle entre opinião e consumo de medicamento por propaganda $(p=$ $0,55)$. A pesar de a associação não ser estatisticamente significante, o dado é relevante e requer maior investigação. Para Bertoldi et al. ${ }^{53}$, um aspecto que induz o uso dos medicamentoséa criação deuma necessidade, em geral, motivada pela propaganda de medicamentos promovida pela indústria farmacêutica.

Em 2004, a Anvisa - por meio da Gerência de M onitoramento e Fiscalização de Propaganda, Publicidade, Promoção e Informação de Produtos Sujeitos à Vigilância Sanitária (GPROP) criou o Projeto de M onitorização da Propaganda e Publicidade de Produtos sob Vigilância Sanitária, visando integrar universidades detodo o Brasil para uma atuação conjunta, no sentido de regulamentar as relações de produção e consumo, garantindo a segurança aos usuários de medicamentos. Porém, de acordo com Soares ${ }^{22}$, todos os estudos sobre propagandas de medicamentos no Brasil vêm mostrando quão ineficaz tem sido a ação das autoridades sanitárias neste setor. Portanto, épreciso delinear novas estratégias queconsolidem o controle da propaganda de forma mais efetiva e que garanta a segurança aos usuários.

\section{Limitações do estudo}

As informações quanto à utilização dos medicamentos podem estar incompletas porque foram coletadas com base nas informações fornecidas pelos usuários. A utilização de outros medicamentos e o seu consumo influenciado pela 
propaganda podem não ter sido relatados por esquecimento dos entrevistados.

\section{Conclusão}

0 incremento da população idosa em termos numéricoseo perfil de morbidade quea caracteriza ( predomínio das condições crônicas) trazem como corolário a preocupação com o aumento no consumo, pelo supramencionado segmento populacional, tanto de medicamentos não prescritos (via automedicação) como, segundo apontam alguns estudos, em maior magnitude, daqueles prescritos. Deforma geral, particularmente nos países desenvolvidos as práticas terapêuticas seguidas pelos médicos regulam, em grande medida, o consumo de medicamentos. Em relação aos idosos, certamente fatores sociais e psicológicos exercem impacto no padrão de uso dos medicamentos: o fato de viver sozinho, sentimentos de solidão ou sintomas de depressão têm sido associados a um consumo aumentado.
No estudo, foi observado que o grupo de idosos que consome medicamentos influenciado pela propaganda não leva em consideração os riscos associados ao uso da farmacoterapia. Portanto, o conhecimento dosfatores queinfluenciam no consumo de medicamentos pela população geriátrica éfundamental para o delineamento de estratégias de utilização racional da farmacoterapia.

Diante disso, é necessário maior aprofundamento no debate sobre a propaganda envolvendo tanto a população quanto profissionais de saúde e órgão reguladores, a fim de avaliar os riscos e ben efícios provenientes do marketing de medicamentos. É deseesperar, igual menteque, a regulamentação da publicidade (RDC n० 102/ 2000, da Anvisa), no momento sob revisão, possa ser aprimorada e vir a impor restrições mais consistentes à ainda imperante liberdade de que gozam os produtores na veiculação, não só pela mídia mas, também, junto aos profissionais de saúde, de peças publicitárias enganosas ou que deixam de apresentar informações cruciais para a boa prescrição e utilização dos medicamentos.

\section{Colaboradores}

AS N eves eKS Cerqueira foram responsáveis pela revisão de literatura, coleta e análise dos dados e redação do artigo; TC M arques e JAC Barros fizeram uma revisão crítica do artigo final; DP Lyra Júnior orientou o trabalho, supervisionou a análise e a redação e fez a revisão crítica do artigo final; PS M arcellini auxiliou na análise estatística e na interpretação dos dados.

\section{Agradecimentos}

Aos profissionais de saúde da Unidade Básica de Saúde Edézio Vieira de M elo, pela colaboração na coleta dos dados, e à professora Chiara Ermínia da Rocha, pela relevante colaboração na revisão final do artigo. 
1. Minayo MCS, Coimbra Jr CEA. Entre a liberdade e a dependência: reflexões sobre o fenômeno social do envelhecimento. In: M inayo MCS, Coimbra Jr CEA, organizadores. Antropologia, saúde e envelhecimento. Rio de Janeiro: Editora Fiocruz; 2002. p. 11-24.

2. U chôa E. Contribuições da antropologia para uma abordagem das questões relativas à saúde do idoso. Cad Saude Publica 2003; 19(3):849-853.

3. Organização das Nações Unidas. População idosa será maior que a de crianças pela primeira vez na história em 2050. U OL 2007; abril 11 [sita da Internet] [acessado 2007 jun 22]. Disponível em: http:// www.noticias.uol.com.br/ultnot/efe/2007/04/11/ ult1766u21157.jhtm

4. Instituto Brasileiro de Geografia e Estatística (IBGE). Síntese de Indicadores Sociais 2004. [site da Internet] [acessado 2007 ago 10]. Disponível em: http:// www.ibge.gov.br/home/presid.php?id_noticia $=132$ \&id_pagina=1

5. Fonseca JE, Carmo TA. O idoso e os medicamentos. Saúde em Revista 2000; 2(4):35-41.

6. Chaimowicz F. A saúde dos idosos brasileiros as vésperas do século XXI: problemas, projeções e alternativas. Rev Saude Publica 1997; 31:184-200.

7. Barros JAC. Políticas farmacêuticas: a serviço dos interesses da saúde? Brasília: Unesco; 2004.

8. Gallo JJ, Whitehead JB, Rabins PV, Murphy JB. Reichel assistência ao idoso: aspectos clínicos do enveIhecimento. Rio de Janeiro: Guanabara Koogan; 2001.

9. Teixeira JJV, Lefèvre F. A prescrição medicamentosa sob a ótica do paciente idoso. Rev Saude Publica 2001; 35(2):207-213.

10. Rozenfeld $\mathrm{S}$. Prevalência, fatores associados e mau uso de medicamentos entre os idosos: uma revisão. Rev Saude Publica 2003; 19(3):717-724.

11. Coelho FJM, Marcopito LF, Castelo A. Perfil de utilização de medicamentos por idosos em área urbana do Nordeste do Brasil. Rev Saude Publica 2004; 38(4):557-564.

12. Nóbrega OT, Karnikowski M GO. A terapia medicamentosa no idoso: cuidados na medicação. Cad Saude Col 2005; 10(2):309-313.

13. Beers M H. Aging as a risk factor for medicationrelated problems. The Consultant Pharmacist (Norfolk) 1999; 14(12):1334-1341.

14. Tapia-Conyer $R$, Cravioto $P$, Borges-Yáñez $A$, De La Rosa B. Consumo de drogas médicas em poblacion de 60 a 65 años en M éxico. Salud Pública de M éxico 1996; 38(6):458-465.

15. Ernest FR, Grizzel AJ. Drug-related morbidity and mortality: updating the cost-of-illness model. Journal of American Pharmaceutical Association 2001; 41:192-199.

16. López MJO. Errores de medicación y gestión de riesgos. Rev Esp Salud Publica 2004; 77:527-540.
17. Loyola Filho Al, Uchoa E. Automedicação: motivações e características de sua prática. Rev M éd M inas Gerais 2002; 12:219-227.

18. Lowe NK, Ryan-Wenger NM . Over-the-counter medications and self-care. Nurse Pract 1999; 24:34-44.

19. Loyola AIF, Uchoa E, Firmo JOA, Lima Costa M F. Estudo de base populacional sobre o consumo de medicamentos entre idosos: Projeto Bambuí. Cad Saude Publica 2005; 21(2):545-553.

20. Lefèvre F. 0 medicamento como mercadoria simbólica. São Paulo: Cortez; 1991.

21. Nascimento AC, Sayd JD. "Ao persistirem os sintomas, o médico deverá ser consultado." Isto é regulação? Rev Saude Colet 2005;15(2):67-75.

22. Soares JCRS. Quando o anúncio é bom, todo mundo compra: o Projeto M onitoração e a propaganda de medicamentos no Brasil. Cien Saude Colet 2008; 13(Sup):641-649.

23. Brasil. Agência Nacional de Vigilância Sanitária (Anvisa). Banco de dados; 2001. [site da Internet] [acessado 2007 abr 24]. Disponível em: http:// www.anvisa.gov.br/medicamentos/legis/resol.htm

24. M assera APD, Camargo JAS, Silva LRFJ. 0 controle do comportamento privado para fins públicos: a monitoração de propaganda de medicamentos no Brasil [monografia]. Brasília: Faculdade de Ciências da Saúde, Universidade de Brasília; 2002.

25. Barros JAC. A (des)informação sobre medicamentos: o duplo padrão de conduta das empresas farmacêuticas. Cad Saude Publica 2000; 16(2):421-427.

26. Lexchin J. Canadian marketing codes: how well are they controlling pharmaceutical promotion? Int J Health Serv 1994; 24:91-104.

27. Herxheimer A, Lundborg CS, Westerholm B. Advertisements for medicines in leading medical journals in 18 countries: a 12-month survey of information content and standards. Int J Health Serv 1993; 23(1):161-172.

28. Silverman $M$, Lee PR, Lydecker $M$. The drugging of the third world. Int J H ealth Serv 1982; 12(4):585-596.

29. Avorn J. Advertising and prescription drugs: promotion, education and the public's health. Health Aff 2003; feb:104-108.

30. Barros JAC. Estratégias mercadológicas da indústria farmacêutica e 0 consumo de medicamentos. Rev Saude Publica 1983; 17:377-386.

31. Pizzol FD, Silva T, Schenkel EP. Análise da adequação das propagandas de medicamentos dirigidas à categoria médica no sul do Brasil. Cad Saude Publica 1998; 14(1):85-91.

32. Fagundes MJD, Soares MGA, Diniz NM, Pires JR, Garrafa V. Análise bioética da propaganda e publicidade de medicamentos. Cien Saude Colet 2007; 12(1):221-229. 
33. M osegui GBG, Rozenfeld S, Veras RP, Vianna CM M Avaliação da qualidade de uso de medicamentos em idosos. Cien Saude Colet 1999; 33(35):437-444.

34. M ontenegro F, Ribeiro VM . 10 Indicador Nacional de Alfabetismo Funcional: um diagnóstico para a inclusão social. São Paulo: Instituto Paulo M ontenegro, Ação Educativa; 2001. [acessado 2007 jun 26]. Disponível em: http://www.acaoeducativa.org. br/downloads/inaf01.pdf

35. Coutinho FL. Avaliação do impacto da intervenção geriátrica na prescrição de idosos. Textos Envelhecimento 2004; 7(2):103-122.

36. Rocha CH, Oliveira APS, Ferreira, C, Faggiani FT, Schroeter G, Souza ACA, De Carli GA, Morrone $\mathrm{FB}$, Werlang $\mathrm{MC}$. Adesão à prescrição médica em idosos de Porto Alegre, RS, Brasil. Cien Saude Colet. 2008, 13(Sup):703-710.

37. Flores LM, M engue SS. U so de medicamentos por idosos em região do Sul do Brasil. Rev Saude Publica 2005; 39(6):924-929.

38. Blanski CR, Lenardt MHA. Compreensão da terapêutica medicamentosa pelo idoso. Nursing Journal of Rio Grande do Sul 2005; 26(2):137-281.

39. Jeckel Neto E, Cunha GL. Teorias biológicas do envelhecimento. In: Vianna EFM, Py L, Neri AL, Cançado FAX, Gorzoni ML, Rocha SM, organizadores. Tratado de geriatria e gerontologia. Rio de Janeiro: Guanabara Koogan; 2002. p. 13-19.

40. Instituto Brasileiro de Geografia e Estatística (IBGE) Síntese de Indicadores Sociais 2006. [site da Internet] [acessado 2007 jun 18]. Disponível em: http:// www.ibge.gov.br/home/presidencia/noticias/ noticia_visualiza.php?id_noticia $=774$

41. Vivian $\overline{E M}$. Improving pressure control in a pharmacist-managed hypertension clinic. Pharmacotherapy 2002; 22(12):1533-1540.

42. Lyra Jr. DP, Amaral RT, Abriata JP, Pelá IR. Satisfacción como resultado de un programa de atención farmacéutica para pacientes ancianos en Ribeirão Preto - São Paulo (Brasil). Seguimiento Farmacoterapêutico 2005; 3(1):30-42.

43. Barros JAC. A medicalização da clientela previdenciária [dissertação]. São Paulo: Faculdade de M edicina da Universidade de São Paulo; 1982.

44. Barros M BA. Saúde e classe social um estudo sobre morbidade e consumo de medicamentos [tese]. Ribeirão Preto: Faculdade de Medicina de Ribeirão Preto da Universidade de São Paulo; 1985.

45. Simões MJS, Farache Filho A. Consumo de medicamentos em região do estado de São Paulo (Brasil), 1985. Rev Saude Publica 1988; 22(6):494-499.
46. Rodrigues RV. O uso indiscriminado de medicamentos pela população de Porto Velho. Anais do II Seminário Integrado de Pesquisa Pós-Graduação e Extensão. XIV Seminário de Iniciação Científica. PI$\mathrm{BIC/UNIR/CN} \mathrm{Pq;} 2005$ [acessado 2007 jun 26]. Disponível em: http://www.unir.br/html/pesquisa/ Pibic_XIV/pibic.2006/artigos.html

47. Revista Saúde Agora. Propaganda de medicamentos versus responsabilidade social da indústria farmacêutica. [acessado 2007 abr 16]. Disponível em: http:/ /www.revistasaudeagora.com.br/htm/materia.asp/ materia $=450$

48. Pinheiro ES. A indústria farmacêutica transnacional e o mercado brasileiro. In: Bonfim JRA, Bermudez JAZ, organizadores. M edicamentos e a reforma do setor saúde. São Paulo: Hucitec, Sobravime; 1999. p. 165-178.

49. Rollason $\mathrm{V}$, Vogt $\mathrm{N}$. Reduction of polypharmacy in the elderly: a systematic review of the role of the pharmacist. Rev Drugs Aging 2003; 20(11):817-832.

50. Lucchese AD, Marçal BF, Araújo GF, Uliana LZ, Rocha MRG, Pinto TJA. Monitoração de propaganda e publicidade de medicamentos: âmbito de São Paulo. Rev Bras Cienc Farm 2005; 41(3):346-349.

51. Heineck I, Gallina SM, Silva T, Pizzol FD, Schenkel EP. Análise da publicidade de medicamentos veiculada em rádios do RS. Cad Saude Publica 1998; 14(1):193-198.

52. Frosch DL, Krueger PM, Hornik RC, Cronholm PF, Barg FK. Creating demand for prescripition drugs: a content analysis of television direct-to-consumer advertising. Rev Annals of Family M edicine 2007; 5(1):6-13.

53. Bertoldi DA, Barros JD, Hallal PC, Lima RC. Utilização de medicamentos em adultos: prevalência e determinantes individuais. Rev Saude Publica 2004; 38(2):228-238.

54. Lopes RGC. Saúde na velhice: as interpretações e os reflexos no uso do medicamento. São Paulo: Educ; 2000.

Artigo apresentado em 07/09/2007

Aprovado em 17/03/2008

Versão final apresentada em 17/04/2008 